\title{
Treatment patterns and clinical outcomes in patients with metastatic breast cancer treated with palbociclib-based therapies: Real-world data in the Han population
}

\section{Hongnan Mo}

Chinese Academy of Medical Sciences Cancer Institute and Hospital https://orcid.org/0000-00022887-7985

Fei Ma ( $\sim$ drmafei@126.com)

Chinese Academy of Medical Sciences Cancer Institute and Hospital https://orcid.org/0000-00019432-1902

\section{Qing Li}

Chinese Academy of Medical Sciences Cancer Institute and Hospital

\section{Pin Zhang}

Chinese Academy of Medical Sciences Cancer Institute and Hospital

\section{Peng Yuan}

Chinese Academy of Medical Sciences Cancer Institute and Hospital

\section{Jiayu Wang}

Chinese Academy of Medical Sciences Cancer Institute and Hospital

\section{Yang Luo}

Chinese Academy of Medical Sciences Cancer Institute and Hospital

\section{Ruigang Cai}

Chinese Academy of Medical Sciences Cancer Institute and Hospital

\section{Qiao Li}

Chinese Academy of Medical Sciences Cancer Institute and Hospital

\section{Binghe Xu}

Chinese Academy of Medical Sciences Cancer Institute and Hospital

\section{Research article}

Keywords: palbociclib, metastatic breast cancer, real-world data, progression-free survival

Posted Date: September 28th, 2020

DOl: https://doi.org/10.21203/rs.3.rs-21850/v2 
License: (c) (i) This work is licensed under a Creative Commons Attribution 4.0 International License. Read Full License 


\section{Abstract}

Background: Palbociclib combined with endocrine therapy has become the standard treatment for estrogen receptor-positive (ER+) metastatic breast cancer. However, little is known about the effectiveness of diverse palbociclib-based regimens other than letrozole and fulvestrant in the real-world clinical setting. This study aimed to reveal the treatment patterns and clinical outcomes in Han patients in routine clinical practice.

Methods: The clinical data of patients with ER+ metastatic breast cancer treated with palbociclib were collected from the China National Cancer Center database. The efficacy profile of palbociclib in this Han population was evaluated, especially in patients younger than 40 years, in those with bone-only metastasis, for various regimen combinations, and as different treatment lines. Propensity score matching was employed to match patients with or without previous everolimus treatment.

Results: A total of 186 patients from 89 cities in 18 provinces in China were enrolled. The median progression-free survival (PFS) was similar among different palbociclib-combined groups $(P=0.566)$ : 10.0 months (95\% confidence interval [Cl] 3.8-16.1) in the exemestane plus palbociclib group, 9.7 months (95\% Cl 6.3-13.1) in the letrozole plus palbociclib group, 7.8 months $(95 \% \mathrm{Cl} 5.5-10.2)$ in the fulvestrant plus palbociclib group, 7.2 months $(95 \% \mathrm{Cl} 3.2-11.3)$ in the toremifene plus palbociclib group, and 6.1 months (95\% $\mathrm{Cl} 1.2-11.0)$ in the anastrozole plus palbociclib group. Kaplan-Meier analysis revealed that patients with bone-only metastasis (median PFS: 8.8 vs. 7.8 months; $P=0.023$ ) and those who received palbociclib as first-line treatment (median PFS: 14.0 months, $95 \% \mathrm{Cl} 11.4-16.6 ; \mathrm{P}<0.001$ ) had prolonged PFS compared with other patients. Patients pretreated with everolimus had significantly worse PFS (3.4 months, 95\% $\mathrm{Cl} 0.7-6.1$ ) than those in the everolimus-naïve group (8.8 months, $95 \% \mathrm{Cl} 6.6$ $11.0, \mathrm{P}=0.001)$ in the whole population. After propensity score matching, patients pretreated with everolimus had inferior PFS (4.4 months, $95 \% \mathrm{Cl} 0.5-8.2)$ compared with everolimus-naïve patients (6.1 months, $95 \% \mathrm{Cl} 4.7-7.5, \mathrm{P}=0.439)$.

Conclusions: Various palbociclib-based regimens have promising efficacy in real-world settings, even in patients with bone-only metastasis. Palbociclib resistance is more common in patients pretreated with everolimus, and in the settings of subsequent treatment compared with first-line treatment.

\section{Background}

Breast cancer is the most diagnosed carcinoma among women in China and worldwide(1), and estrogen receptor-positive (ER+) breast cancer is the most common subtype(2). In these patients, significant advantages for survival were observed for cyclin-dependent kinases 4 and 6 (CDK4/6) inhibitors, including palbociclib, ribociclib, and abemaciclib(3). In the settings of clinical trials, palbociclib in combination with fulvestrant or letrozole as first-line treatment has significantly prolonged progressionfree survival (PFS) in ER+ metastatic breast cancer patients from 6.6 to 10.3 months with tolerable side effects $(4,5)$. Real-world data from the United States, mainly of White people, have supported the benefits 
of the addition of CDK4/6 inhibitors as first-line treatment for improving long-term outcomes(6-8). Data from other races may further support the value of CDK4/6 inhibitors in patients with ER+ metastatic breast cancer.

Preclinical and clinical studies have suggested that CDK6 amplification, cyclin E1 (CCNE1) amplification, and $R B 1$ mutation may result in resistance to CDK4/6 inhibition(9-11). Recently, studies of the mechanisms of resistance to CDK4/6 inhibitors have focused on the phosphatidylinositol-3-kinase/Akt (PIK3CA/Akt) and mammalian target of rapamycin (mTOR) pathway. Upregulated PI3K/Akt/mTOR signal has been found in response to chronic exposure to CDK4/ 6 inhibitors which bypasses the CDK4/ 6 axis and subsequently drives cell cycle progression(12). Liquid biopsy in the PALOMA-3 trial found that new driver mutations emerged in PIK3CA after palbociclib plus fulvestrant treatment(13). Notably, the PALOMA-3 trial excluded patients who had previously received everolimus, an mTOR inhibitor. Thus, the clinical outcomes and toxicity data of CDK4/6 inhibitor combinations in patients previously treated with mTOR inhibitors are poorly understood.

Here, we conducted a cohort study in patients with ER+ metastatic breast cancer using the China National Cancer Center database. The efficacy profile of palbociclib (the only available CDK4/6 inhibitor in China) in the real-world setting in the Han population was investigated. Furthermore, the clinical outcomes of palbociclib-based treatment in patients with prior exposure to everolimus (mTOR inhibitor; PI3K/Akt inhibitors are not available in China at present) were compared to those of everolimus-naïve patients.

\section{Methods}

\section{Study design and patient population}

The medical charts of patients who were prescribed palbociclib from May 1, 2016 to November 30, 2019 were collected from the database of the China National Cancer Center and reviewed. Patients were included if they 1) were diagnosed with metastatic breast carcinoma, 2) had confirmed ER+ human epidermal growth factor receptor 2-negative (HER2-) tumors, 3) received palbociclib-containing treatment, and 4) completed at least 1 month of follow-up after the prescription. The clinical and pathological characteristics of the patients were analyzed. The ER and Ki-67 status were evaluated based on metastatic biopsy results, except for those with bone-only metastases, which were evaluated based on the primary lesion. The efficacy profile of palbociclib in this Han population was evaluated, especially in patients younger than 40 years, in those with bone-only metastasis, as different treatment lines, and for various combination regimens. The efficacy of palbociclib-based therapy in patients with prior everolimus treatment was also assessed. The study was authorized by the review board of the Cancer Hospital, Chinese Academy of Medical Sciences (19/331-2115), and all patients signed informed consents before treatment.

\section{Statistical analysis}


Patients' baseline characteristics were categorized and compared using $\chi^{2}$ tests. Visceral disease was defined as metastatic breast cancer with visceral organ involvement (lung, liver, peritoneum or pleura) that was present at the initiation of palbociclib treatment. A propensity score for previously receiving everolimus was estimated using logistic regression with the following covariates (Table 1): age, stage at diagnosis, progesterone receptor-positive rate, $\mathrm{Ki}-67$ positive rate, and previous lines of treatment.

The disease control rate (DCR) was defined as the percentage of patients who achieved complete response, partial response, or stable disease after treatment. PFS was defined as the time from the initiation of palbociclib to the date of disease progression which was determined by the physician based on available radiologic information, hematologic tumor markers, and/or clinical information. The KaplanMeier method and log-rank test were used to estimate and compare survival curves, both in the original population and in the matched population according to the propensity scores. Cox regression analysis was applied to identify independent predictors of survival in the multivariate analysis. A two-sided $P$ value $<0.05$ was considered statistically significant. All statistical analyses were carried out using IBM SPSS version 24.0 software (IBM Corp., Armonk, NY), including propensity-score matching.

\section{Results}

\section{Patient characteristics}

From May 1, 2016, to November 30, 2019, a total of 186 patients were enrolled in this study (Figure 1). Patients were from 89 cities in 18 provinces in China. The greatest number of patients come from five regions: Beijing (74, 42.5\%), Hebei (28, 15.1\%), Shandong (15, 8.1\%), Inner Mongolia (13, 7.0\%), and Heilongjiang $(11,5.9 \%)$. The median age was 54 years (range $28-90)$, and 29 patients $(15.6 \%)$ were younger than 40 years. Thirty-three patients $(17.7 \%)$ had de novo metastatic breast cancer. Hundred thirty-eight (74.2\%) patients had visceral disease when they started the palbociclib therapy, while 16 (8.6\%) patients had bone-only metastasis. Hundred forty-four out of 186 patients $(77.4 \%)$ showed sensitivity to prior endocrine therapy. Patients received distinct endocrine therapy combined with palbociclib: 96 with fulvestrant, 45 with letrozole, 20 with exemestane, 14 with anastrozole, nine with toremifene, and two with medroxyprogesterone. Only 48 patients $(25.8 \%)$ received palbociclib as first-line treatment for their metastatic disease, while 82 patients (44.1\%) had previously undergone at least three lines of systemic treatment. Thirty-four patients (18.3\%) had received everolimus for their metastatic disease before the prescription of palbociclib. The basic clinical and pathological characteristics are detailed in Table 1.

\section{Efficacy profile of palbociclib in the Han population}

As of February 2020, the median follow-up time was 6.5 months (range 0.9-40.0), and 88 patients had disease progression. Univariate analysis revealed that patients who were younger than 40 years $(P=0.022)$, those with metastasis other than bone metastasis $(P=0.069)$, and those who had received 
previous lines of systemic treatment $(P<0.001)$ had significantly worse PFS, while various treatment combinations ( $P=0.763)$, number of metastatic sites $(P=0.508)$, and disease stage at diagnosis $(P=0.620)$ did not influence PFS. Multivariate analysis (Table 2) showed that age and previous lines of systemic treatment before palbociclib-based therapy were both independent factors for PFS $(P=0.027$ and $P<0.001$, respectively).

The DCR of palbociclib-based therapy varied significantly among different age groups $(P=0.034)$, i.e., the DCR was $60.0 \%(20 / 29)$ in patients younger than 40 years, $77.1 \%(108 / 140)$ in those aged $40-70$ years, and $82.3 \%(14 / 17)$ in patients older than 70 years. Accordingly, patients younger than 40 years had a worse PFS (4.0 months, $95 \%$ confidence interval [Cl] 0-8.5) than patients older than 40 years $(8.4$ months in those aged $40-70$ years, and 6.2 months in those older than 70 years, $P=0.189$, Figure 2A). The DCR after palbociclib-based treatment was promising in patients with bone-only metastasis $(93.7 \% \mathrm{vs}$. $74.7 \% \%, P=0.087)$, and these patients also had significantly prolonged PFS compared with the other patients ( 8.8 vs. 7.8 months, $P=0.023$, Figure $2 \mathrm{~B}$ ).

The efficacy of palbociclib-based treatment changed in accordance with increasing numbers of previous lines of systemic treatment (Figure $2 \mathrm{C}$ ). As the number of previous lines of systemic treatment increased, the DCR gradually decreased $(P=0.001)$, i.e., the DCR was $93.7 \%(45 / 48)$ in the setting of first-line treatment, $80.3 \%(45 / 56)$ in patients with one to two prior regimens, $65.9 \%(29 / 44)$ in patients with three to four prior regimens, and $60.5 \%(23 / 38)$ in patients who had been treated with more than five regimens. The median PFS was also longer in patients who had received palbociclib-based therapy as first-line treatment (14.0 months, $95 \% \mathrm{Cl} 11.4-16.6)$ than in those who received subsequent lines of treatment $(P<0.001)$, i.e., 10.0 months ( $95 \% \mathrm{Cl} 7.1-12.9)$ in the second/third-line group, 6.2 months $(95 \% \mathrm{Cl} 3.0-$ $9.5)$ in the fourth/fifth-line group, and 3.4 months $(95 \% \mathrm{Cl} 0.8-6.1)$ in patients who had received more than five lines of systemic therapy.

The DCR did not differ significantly among patients who received diverse palbociclib-based treatment $(P=0.403)$, i.e., the DCR was $86.7 \%(39 / 45)$ in the letrozole-combined group, $74.0 \%(71 / 96)$ in the fulvestrant-combined group, $71.4 \%(10 / 14)$ in the anastrozole-combined group, $70.0 \%(14 / 20)$ in the exemestane-combined group, and $66.7 \%$ in the toremifene-combined group (6/9). The median PFS was also similar among different palbociclib-combined groups ( $P=0.566$, Figure $2 D)$, i.e., 10.0 months $(95 \% \mathrm{Cl}$ 3.8-16.1) in the exemestane plus palbociclib group, 9.7 months $(95 \% \mathrm{Cl} 6.3-13.1)$ in the letrozole plus palbociclib group, 7.8 months $(95 \% \mathrm{Cl} 5.5-10.2)$ in the fulvestrant plus palbociclib group, 7.2 months (95\% $\mathrm{Cl} 3.2-11.3)$ in the toremifene plus palbociclib group, and 6.1 months ( $95 \% \mathrm{Cl} 1.2-11.0)$ in the anastrozole plus palbociclib group. One of the two patients receiving medroxyprogesterone plus palbociclib had stable disease for more than 12 months; however, palbociclib treatment was interrupted for financial reasons. The other patient experienced disease progression after 2.5 months of treatment with medroxyprogesterone plus palbociclib.

\section{Efficacy in patients with prior everolimus treatment}


Regarding the previous treatment before palbociclib-based therapy, everolimus was mostly combined with exemestane $(15 / 34,44.1 \%)$, followed by fulvestrant $(7 / 34,20.1 \%)$, and toremifene $(6 / 34,17.6 \%)$.

The DCR was significantly lower in patients who had received previous everolimus $(50.0 \%, 17 / 34)$ than in the everolimus-naïve group $(82.2 \%, 125 / 152, P<0.001)$. Consistently, the Kaplan-Meier estimates indicated that patients pretreated with everolimus had significantly worse PFS (3.4 months, 0.7-6.1) than patients in the everolimus-naïve group ( 8.8 months, $95 \% \mathrm{Cl} 6.6-11.0, P=0.001$, Figure $3 \mathrm{~A}$ ). Further, propensity score matching was used to match patients with or without prior everolimus treatment. Propensity score matching resulted in 30 patients in the previous-everolimus group and 30 patients in the everolimus-naïve group (Table 1). After propensity score matching, no significant differences in the clinical characteristics were observed between the two groups. Palbociclib-based therapy resulted in a worse DCR of $53.3 \%(16 / 30)$ in the previous-everolimus group compared with $76.7 \%(23 / 30)$ in the everolimus-naïve group $(P=0.058)$. Consistently, patients pretreated with everolimus had inferior PFS (4.4 months, $95 \% \mathrm{Cl} 0.5-8.2)$ than everolimus-naïve patients (6.1 months, $95 \% \mathrm{Cl} 4.7-7.5, P=0.439$, Figure $3 \mathrm{~B})$.

\section{Discussion}

To our knowledge, this is the first real-world assessment of the efficacy of palbociclib in the Han population that reveals the treatment patterns for 186 Chinese patients from 89 major cities. We found that palbociclib-based therapy as first-line systemic treatment resulted in a DCR of $93.7 \%$ and a median PFS of 14.0 months in Han patients with ER+HER2- metastatic breast cancer. The efficacy profile of palbociclib-based treatment in the Han population was similar to the real-world clinical outcomes in patients from the United States $(6,8,14)$. These results are also comparable to the clinical benefit rate (CBR) and PFS data from the PALOMA-2 (palbociclib plus letrozole, CBR 84.3\%, median PFS 24.8 months) and PALOMA-3 (palbociclib plus fulvestrant, CBR 67\%, median PFS 9.5 months) studies(4, 5, 15). Thus, palbociclib-based combinations have promising efficacy for patients with ER+HER2metastatic breast cancer in the Han population.

Our study deepens the understanding of the use of palbociclib-based treatments and related clinical outcomes in the real-world. We found that the clinical efficacy of the various palbociclib-based regimens was similar, including exemestane plus palbociclib, and toremifene plus palbociclib regimens. Thus, endocrine therapy in combination with palbociclib may not be limited to fulvestrant and letrozole. Exemestane or toremifene in combination with palbociclib may be considered as an option in clinical trials for patients who are resistant to fulvestrant and letrozole after previous systemic treatment. Several clinical trials have been launched to evaluated the efficacy of exemestane plus palbociclib in patients with metastatic breast cancer (NCT02871791, NCT02592746).

Significant differences between the real-world patients and those in clinical trials receiving palbociclib should be considered. We included patients with bone-only metastasis who lacked measurable lesions. These patients are not uncommon in clinical settings; however, they are ineligible for clinical trials 
because of a lack of target lesions. In these patients, palbociclib-based combinations resulted in a DCR of 93.7\% and median PFS of 8.8 months. Moreover, we enrolled patients who had undergone intensive treatment with more than five lines of systemic treatment; these patients had a terrible median PFS of 3.4 months after palbociclib-based combinations. ,These patients comprise a considerable proportion of the patient population in clinical practice; however, they are usually underrepresented in clinical trials. Since CDK4/6 inhibitors are not covered by medical insurance in China, a substantial proportion of patients cannot afford these drugs. Therefore, it is recommended that clinicians consider the economic benefit ratio in the future and take care to avoid using palbociclib-based regimens in these heavily pretreated patients.

Some patients have an innate primary resistance to CDK4/6 inhibitors(16). The assessment of the predictive ability of clinical indicators for drug resistance could provide a reference when planning a patient's medication regimen. Here, we found that palbociclib as first-line treatment resulted in improved efficacy compared with its use during subsequent lines of treatment. Notably, patients younger than 40 years tended to experience more malignant disease and worse prognosis, even if they had undergone palbociclib-based treatment.

As several preclinical studies have shown that the activation of the PI3K/Akt/mTOR pathway had an impact on the resistance to CDK4/6 inhibitors $(12,13,17)$, we analyzed patients based on whether or not they had received previous everolimus treatment. In the whole population, we found that patients who had been treated with everolimus had significantly worse DCR (50.0\%) and PFS (3.4 months) than everolimus-naïve patients. This finding appears to be consistent with that of a retrospective study of 23 patients in the United States(18). In that study, Dhakal et al. found that palbociclib-based therapy had a DCR of $17.4 \%$ and a median PFS of 2.9 months in patients with metastatic breast cancer pretreated with everolimus(18). In contrast, a prospective single-arm clinical trial in France that evaluated the efficacy of palbociclib plus fulvestrant in patients pretreated with everolimus reported a DCR of $71.7 \%$ and median PFS of 5.8 months(19). Thus, we used propensity score matching to reduce the interference of other variables such as previous lines of treatment. Subsequently, the difference between the patients with or without prior everolimus became less significant.

The limitations of our study should be considered. Our data originated from the retrospective review of medical charts in a single institution. However, the database of the China National Cancer Center covers most of the provinces in China which reduces potential bias. This study included patients who received palbociclib between 2016 and 2019. The drug was officially approved for marketing in mainland China in 2018 and has not been covered by medical insurance. Therefore, the number of participants included in this study is limited. Only patients who could afford the drug received palbociclib, which may be a potential source of bias. Moreover, the evaluation of the clinical response did not involve independent radiological confirmation. Thus, our results should be interpreted with caution.

\section{Conclusion}


This real-world analysis revealed the treatment patterns and clinical outcomes after palbociclib-based combinations for ER+HER2- metastatic breast cancer in the Han population. Our results demonstrated the promising efficacy of various regimens combined with palbociclib in real-world settings, even in patients with bone-only metastasis. Exemestane or toremifene could also be considered in combination with palbociclib in patients who are resistant to fulvestrant and letrozole after previous systemic treatment. Palbociclib resistance was more common in patients pretreated with everolimus, and in the settings of subsequent lines of treatment compared with first-line treatment. Further studies with larger sample sizes and longer follow-up are warranted to confirm our findings.

\section{Abbreviations}

estrogen receptor-positive (ER+)

cyclin-dependent kinases 4 and 6 (CDK4/6)

progression-free survival (PFS)

phosphatidylinositol-3-kinase/Akt (PIK3CA/Akt)

mammalian target of rapamycin (mTOR)

human epidermal growth factor receptor 2-negative (HER2-)

disease control rate (DCR)

confidence interval $(\mathrm{Cl})$

clinical benefit rate (CBR)

\section{Declarations}

\section{Ethics approval and consent to participate}

All procedures performed in studies involving human participants were in accordance with the ethical standards of the institutional and/or national research committee and with the 1964 Helsinki Declaration and its later amendments or comparable ethical standards. The study was approved by the review board of the Cancer Hospital, Chinese Academy of Medical Sciences (19/331-2115).

Informed consent was obtained from all individual participants included in the study.

\section{Consent for publication}

Not applicable 
The datasets generated and/or analysed during the current study are not publicly available due to the rules of the institution, but are available from the corresponding author on reasonable request.

\section{Competing interests}

The authors declare that they have no competing interests.

\section{Funding}

This work was funded by grants from National Natural Science Foundation of China (81902705) and Beijing Natural Science Foundation (7204292). The funding bodies have no role in the design of the study and collection, analysis and interpretation of data. They were not involved in writing the manuscript.

\section{Author contributions}

HM and FM conceived the study, collected and analyzed the data, interpreted the results and wrote the manuscript. QingL, PZ, PY, JW and BX made substantial contributions to the conception of the study, performed experiments, analyzed data, and was substantially involved in writing and revising the manuscript. YL was involved in data acquisition and data analysis. RC and QiaoL conceived the study, designed the experiments, analyzed the data, interpreted the results, wrote the manuscript and revision of the manuscript, and provided financial support. All authors have read and approved the final manuscript.

\section{Acknowledgements}

Not applicable

\section{References}

1. Bray F, Ferlay J, Soerjomataram I, Siegel RL, Torre LA, Jemal A. Global cancer statistics 2018 : GLOBOCAN estimates of incidence and mortality worldwide for 36 cancers in 185 countries. CA: a cancer journal for clinicians. 2018;68(6):394-424.

2. Howlader N, Altekruse SF, Li Cl, Chen VW, Clarke CA, Ries LAG, et al. US Incidence of Breast Cancer Subtypes Defined by Joint Hormone Receptor and HER2 Status. JNCl: Journal of the National Cancer Institute. 2014;106(5).

3. Li J, Fu F, Yu L, Huang M, Lin Y, Mei Q, et al. Cyclin-dependent kinase 4 and 6 inhibitors in hormone receptor-positive, human epidermal growth factor receptor-2 negative advanced breast cancer: a meta-analysis of randomized clinical trials. Breast cancer research and treatment. 2020;180(1):2132.

4. Finn RS, Martin M, Rugo HS, Jones S, Im SA, Gelmon K, et al. Palbociclib and Letrozole in Advanced Breast Cancer. The New England journal of medicine. 2016;375(20):1925-36. 
5. Turner NC, Slamon DJ, Ro J, Bondarenko I, Im SA, Masuda N, et al. Overall Survival with Palbociclib and Fulvestrant in Advanced Breast Cancer. The New England journal of medicine. 2018;379(20):1926-36.

6. DeMichele A, Cristofanilli M, Brufsky A, Liu X, Mardekian J, McRoy L, et al. Abstract P1-19-02: Overall survival for first-line palbociclib plus letrozole vs letrozole alone for HR+/HER2- metastatic breast cancer patients in US real-world clinical practice. Cancer research. 2020;80(4 Supplement):P1-19-02P1-19-02.

7. Downer M, Jhaveri K, Bardia A, Loi S, Kent M, Luhn P, et al. Abstract P5-11-07: Real-world predictors of first-line treatment and descriptive outcomes in HR<sup $>+</$ sup $>/$ HER2 $<$ sup $>-</$ sup $>$ metastatic breast cancer patients in the US. Cancer research. 2020;80(4 Supplement):P5-11-07-P5-11-07.

8. Fountzilas E, Koliou GA, Rapti V, Nikolakopoulos A, Christopoulou A, Moirogiorgou E, et al. Clinical outcome and toxicity data in patients with advanced breast cancer treated with cyclin-dependent kinase 4/6 (CDK4/6) inhibitors combined with endocrine therapy in a real-world clinical setting. Annals of Oncology. 2019;30:v118.

9. Condorelli R, Spring L, O'Shaughnessy J, Lacroix L, Bailleux C, Scott V, et al. Polyclonal RB1 mutations and acquired resistance to CDK $4 / 6$ inhibitors in patients with metastatic breast cancer. Annals of oncology : official journal of the European Society for Medical Oncology. 2018;29(3):640-5.

10. Turner NC, Liu Y, Zhu Z, Loi S, Colleoni M, Loibl S, et al. Cyclin E1 Expression and Palbociclib Efficacy in Previously Treated Hormone Receptor-Positive Metastatic Breast Cancer. Journal of clinical oncology : official journal of the American Society of Clinical Oncology. 2019:Jco1800925.

11. Yang C, Li Z, Bhatt T, Dickler M, Giri D, Scaltriti M, et al. Acquired CDK6 amplification promotes breast cancer resistance to CDK4/6 inhibitors and loss of ER signaling and dependence. Oncogene. 2017;36(16):2255-64.

12. Herrera-Abreu MT, Palafox M, Asghar U, Rivas MA, Cutts RJ, Garcia-Murillas I, et al. Early Adaptation and Acquired Resistance to CDK4/6 Inhibition in Estrogen Receptor-Positive Breast Cancer. Cancer research. 2016;76(8):2301-13.

13. O'Leary B, Cutts RJ, Liu Y, Hrebien S, Huang X, Fenwick K, et al. The Genetic Landscape and Clonal Evolution of Breast Cancer Resistance to Palbociclib plus Fulvestrant in the PALOMA-3 Trial. Cancer discovery. 2018;8(11):1390-403.

14. Taylor-Stokes G, Mitra D, Waller J, Gibson K, Milligan G, lyer S. Treatment patterns and clinical outcomes among patients receiving palbociclib in combination with an aromatase inhibitor or fulvestrant for HR+/HER2-negative advanced/metastatic breast cancer in real-world settings in the US: Results from the IRIS study. The Breast. 2019;43:22-7.

15. Cristofanilli M, Turner NC, Bondarenko I, Ro J, Im SA, Masuda N, et al. Fulvestrant plus palbociclib versus fulvestrant plus placebo for treatment of hormone-receptor-positive, HER2-negative metastatic breast cancer that progressed on previous endocrine therapy (PALOMA-3): final analysis of the multicentre, double-blind, phase 3 randomised controlled trial. The Lancet Oncology. 2016;17(4):425-39. 
16. McCartney A, Migliaccio I, Bonechi M, Biagioni C, Romagnoli D, De Luca F, et al. Mechanisms of Resistance to CDK4/ 6 Inhibitors: Potential Implications and Biomarkers for Clinical Practice. Frontiers in oncology. 2019;9:666.

17. Jansen VM, Bhola NE, Bauer JA, Formisano L, Lee KM, Hutchinson KE, et al. Kinome-Wide RNA Interference Screen Reveals a Role for PDK1 in Acquired Resistance to CDK4/6 Inhibition in ERPositive Breast Cancer. Cancer research. 2017;77(9):2488-99.

18. Dhakal A, Matthews CM, Levine EG, Salerno KE, Zhang F, Takabe K, et al. Efficacy of Palbociclib Combinations in Hormone Receptor\&\#x2013;Positive Metastatic Breast Cancer Patients After Prior Everolimus Treatment. Clinical breast cancer. 2018;18(6):e1401-e5.

19. du Rusquec P, Palpacuer C, Campion L, Patsouris A, Augereau P, Gourmelon C, et al. Efficacy of palbociclib plus fulvestrant after everolimus in hormone receptor-positive metastatic breast cancer. Breast cancer research and treatment. 2018;168(2):559-66.

\section{Tables}

Table 1. Clinical and pathological characteristics before and after matching on the propensity score. 


\begin{tabular}{|c|c|c|c|c|c|c|c|}
\hline & & $\overline{\mathrm{Be}}$ & re matching & & Aft & matching & \\
\hline & $\begin{array}{c}\text { All } \\
(\mathrm{n}=186)\end{array}$ & $\begin{array}{l}\text { Previous } \\
\text { mTORi } \\
(\mathrm{n}=34)\end{array}$ & $\begin{array}{l}\text { mTORi- } \\
\text { naïve } \\
(\mathrm{n}=152)\end{array}$ & $P$ & $\begin{array}{l}\text { Previous } \\
\text { mTORi } \\
(\mathrm{n}=30)\end{array}$ & $\begin{array}{l}\text { mTORi- } \\
\text { naïve } \\
(\mathrm{n}=30)\end{array}$ & $P$ \\
\hline $\begin{array}{l}\text { Age, n (\%) } \\
<40 \\
40-69 \\
\geq 70\end{array}$ & $\begin{array}{l}29 \\
(15.6 \%) \\
140 \\
(75.3 \%) \\
17 \\
(9.1 \%)\end{array}$ & $\begin{array}{l}4(11.8 \%) \\
29(85.3 \%) \\
1(2.9 \%)\end{array}$ & $\begin{array}{l}25 \\
(16.4 \%) \\
111 \\
(73.0 \%) \\
16 \\
(10.6 \%)\end{array}$ & 0.260 & $\begin{array}{l}3(10.0 \%) \\
27 \\
(90.0 \%) \\
0\end{array}$ & $\begin{array}{l}3 \\
(10.0 \%) \\
25 \\
(83.3 \%) \\
2(6.7 \%)\end{array}$ & 0.354 \\
\hline $\begin{array}{l}\text { Stage at diagnosis, } \mathrm{n} \\
(\%) \\
\text { I-III } \\
\text { IV }\end{array}$ & $\begin{array}{l}153 \\
(82.3 \%) \\
33 \\
(17.7 \%)\end{array}$ & $\begin{array}{l}26(76.5 \%) \\
8(23.5 \%)\end{array}$ & $\begin{array}{l}127 \\
(83.6 \%) \\
25 \\
(16.4 \%)\end{array}$ & 0.328 & $\begin{array}{l}24 \\
(80.0 \%) \\
6(20.0 \%)\end{array}$ & $\begin{array}{l}24 \\
(80.0 \%) \\
6 \\
(20.0 \%)\end{array}$ & 1.000 \\
\hline $\begin{array}{l}* \mathrm{ER}+, \mathrm{n}(\%) \\
1 \%-9 \% \\
\geq 10 \%\end{array}$ & $\begin{array}{l}4(2.2 \%) \\
182 \\
(97.8 \%)\end{array}$ & $\begin{array}{l}3(8.8 \%) \\
31(91.2 \%)\end{array}$ & $\begin{array}{l}1(0.7 \%) \\
151 \\
(99.3 \%)\end{array}$ & 0.003 & $\begin{array}{l}1(3.3 \%) \\
29 \\
(96.7 \%)\end{array}$ & $\begin{array}{l}1(3.3 \%) \\
29 \\
(96.7 \%)\end{array}$ & 1.000 \\
\hline $\begin{array}{l}* \mathrm{PR}+, \mathrm{n}(\%) \\
1 \%-9 \% \\
\geq 10 \%\end{array}$ & $\begin{array}{l}62 \\
(33.3 \%) \\
124 \\
(66.7 \%)\end{array}$ & $\begin{array}{l}14(41.2 \%) \\
20(58.8 \%)\end{array}$ & $\begin{array}{l}48(31.6) \\
104 \\
(68.4 \%)\end{array}$ & 0.283 & $\begin{array}{l}14 \\
(46.7 \%) \\
16 \\
(53.3 \%)\end{array}$ & $\begin{array}{l}10 \\
(33.3 \%) \\
20 \\
(66.7 \%)\end{array}$ & 0.292 \\
\hline $\begin{array}{l}* \mathrm{Ki}-67+, \mathrm{n}(\%) \\
0-13 \% \\
\geq 14 \%\end{array}$ & $\begin{array}{l}25 \\
(13.4 \%) \\
161 \\
(86.6 \%)\end{array}$ & $\begin{array}{l}6(17.6 \%) \\
28(82.4 \%)\end{array}$ & $\begin{array}{l}19 \\
(12.5 \%) \\
133 \\
(87.5 \%)\end{array}$ & 0.426 & $\begin{array}{l}6(20.0 \%) \\
24(80 \%)\end{array}$ & $\begin{array}{l}4 \\
(13.3 \%) \\
26 \\
(86.7 \%)\end{array}$ & 0.488 \\
\hline $\begin{array}{l}\text { Visceral disease, n (\%) } \\
\text { Yes } \\
\text { No }\end{array}$ & $\begin{array}{l}138 \\
(74.2 \%) \\
48 \\
(25.8 \%)\end{array}$ & $\begin{array}{l}28(82.4 \%) \\
6(17.6 \%)\end{array}$ & $\begin{array}{l}110 \\
(72.4 \%) \\
42 \\
(27.6 \%)\end{array}$ & 0.229 & $\begin{array}{l}25 \\
(83.3 \%) \\
5(16.7 \%)\end{array}$ & $\begin{array}{l}26 \\
(86.7 \%) \\
4 \\
(13.3 \%)\end{array}$ & 0.718 \\
\hline $\begin{array}{l}\text { Bone-only disease, n (\%) } \\
\text { Yes } \\
\text { No }\end{array}$ & $\begin{array}{l}16 \\
(8.6 \%) \\
170 \\
(91.4 \%)\end{array}$ & $\begin{array}{l}1(2.9 \%) \\
33(97.1 \%)\end{array}$ & $\begin{array}{l}15(9.9 \%) \\
137 \\
(90.1 \%)\end{array}$ & 0.193 & $\begin{array}{l}1(3.3 \%) \\
29 \\
(96.7 \%)\end{array}$ & $\begin{array}{l}1(3.3 \%) \\
29 \\
(96.7 \%)\end{array}$ & 1.000 \\
\hline $\begin{array}{l}\text { Number of metastatic } \\
\text { sites, n (\%) } \\
1-2 \\
3-4 \\
5-6\end{array}$ & $\begin{array}{l}114 \\
(61.3 \%) \\
64 \\
(34.4 \%) \\
8(4.3 \%)\end{array}$ & $\begin{array}{l}18(52.9 \%) \\
14(41.2 \%) \\
2(5.9 \%)\end{array}$ & $\begin{array}{l}96 \\
(63.2 \%) \\
50 \\
(32.9 \%) \\
6(3.9 \%)\end{array}$ & 0.530 & $\begin{array}{l}17 \\
(56.7 \%) \\
13 \\
(43.3 \%) \\
0\end{array}$ & $\begin{array}{l}17 \\
(56.7 \%) \\
11 \\
(36.7 \%) \\
2(6.6 \%)\end{array}$ & 0.338 \\
\hline $\begin{array}{l}\text { Previous lines of } \\
\text { therapy, n (\%) } \\
0 \\
1-2 \\
3-4 \\
\geq 5\end{array}$ & $\begin{array}{l}48 \\
(25.8 \%) \\
56 \\
(30.1 \%) \\
44 \\
(23.7 \%) \\
38 \\
(20.4 \%) \\
\end{array}$ & $\begin{array}{l}0 \\
6(17.6 \%) \\
8(23.5 \%) \\
20(58.8 \%)\end{array}$ & $\begin{array}{l}48 \\
(31.6 \%) \\
50(32.9 \%) \\
36 \\
(23.7 \%) \\
18 \\
(11.8 \%)\end{array}$ & $<0.001$ & $\begin{array}{l}0 \\
6(20.0 \%) \\
8(26.7 \%) \\
16 \\
(53.3 \%)\end{array}$ & $\begin{array}{l}1(3.3 \%) \\
4 \\
(13.3 \%) \\
9 \\
(30.0 \%) \\
16 \\
(53.3 \%)\end{array}$ & 0.692 \\
\hline $\begin{array}{l}* \mathrm{ER}, \mathrm{PR} \text {, and Ki- } 67 \text { stat } \\
\text { only metastases, which } \\
\text { Abbreviations: ER, es } \\
\text { progesterone receptor }\end{array}$ & evalu & or; mTO & nrimary & 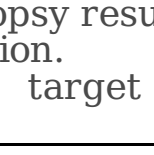 & C & & one- \\
\hline
\end{tabular}


Table 2. Multivariate analysis of PFS in patients treated with palbociclib-based therapy.

\begin{tabular}{|c|c|c|}
\hline Variable & Hazard Ratio (95\% CI) & $P$ \\
\hline Age & & 0.038 \\
\hline$<40$ years old & 1.0 & \\
\hline $40-70$ years old & 0.50 (0.28-0.87) & 0.014 \\
\hline$\geq 70$ years old & $0.76(0.32-1.79)$ & 0.526 \\
\hline Previous lines of treatment & & $<0.001$ \\
\hline 0 & 1.0 & \\
\hline $1-2$ & $2.03(0.97-4.23)$ & 0.060 \\
\hline $3-4$ & $2.77(1.33-5.72)$ & 0.006 \\
\hline$\geq 5$ & $5.10(2.48-10.50)$ & $<0.001$ \\
\hline *Bone-only metastasis & $0.36(0.11-1.18)$ & 0.091 \\
\hline $\begin{array}{l}\text { *The control group includ } \\
\text { other locations, including } \\
\text { Abbreviations: CI, confide }\end{array}$ & $\begin{array}{l}\text { astases, as well as pat } \\
\text { and visceral metastasi } \\
\text { PFS, progression-free }\end{array}$ & tastases in \\
\hline
\end{tabular}

\section{Figures}

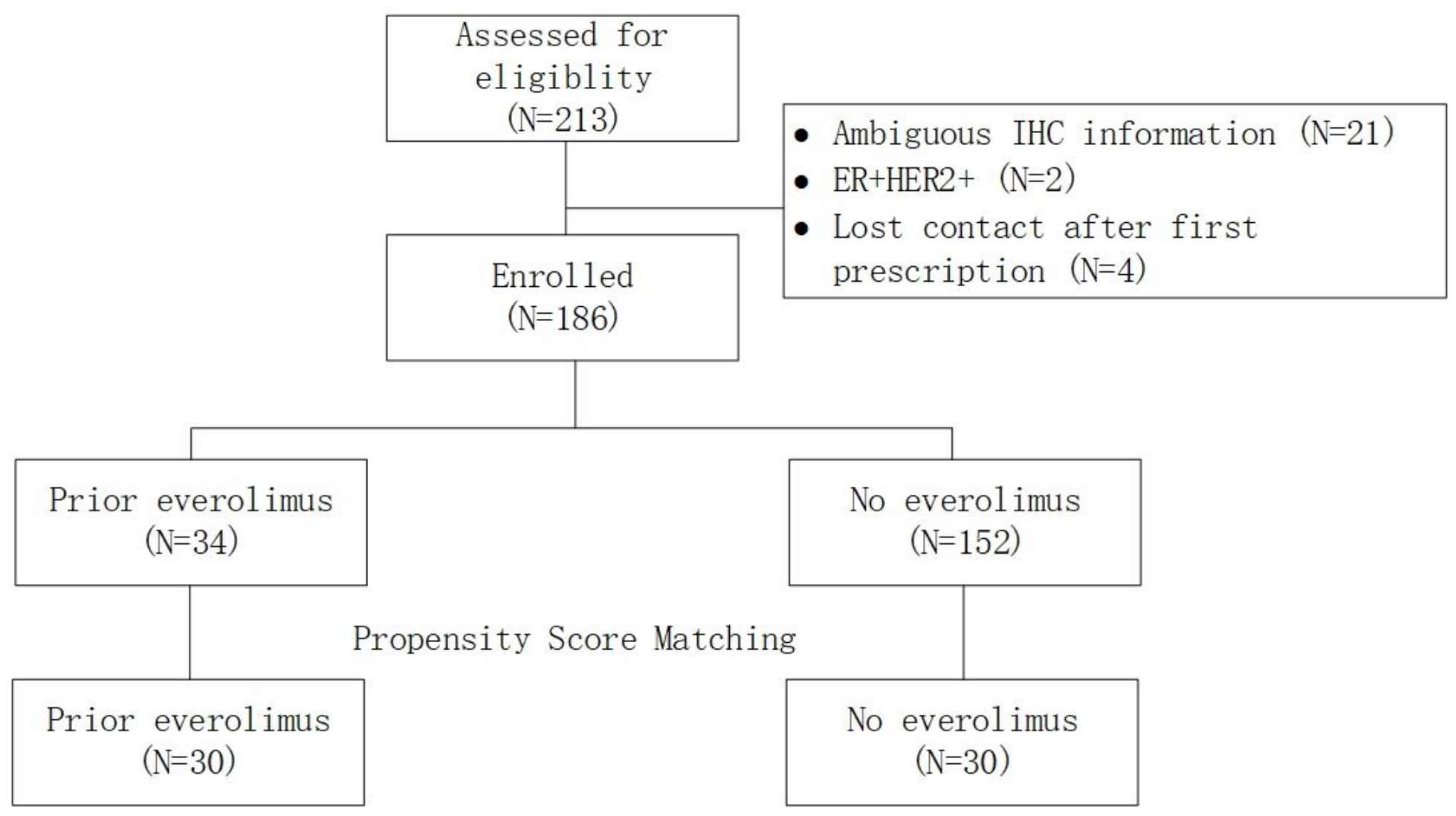

\section{Figure 1}

Patient flow diagram. ER+, estrogen receptor-positive; HER2+, human epidermal growth factor receptor 2positive; IHC, immunohistochemistry. 
(A)

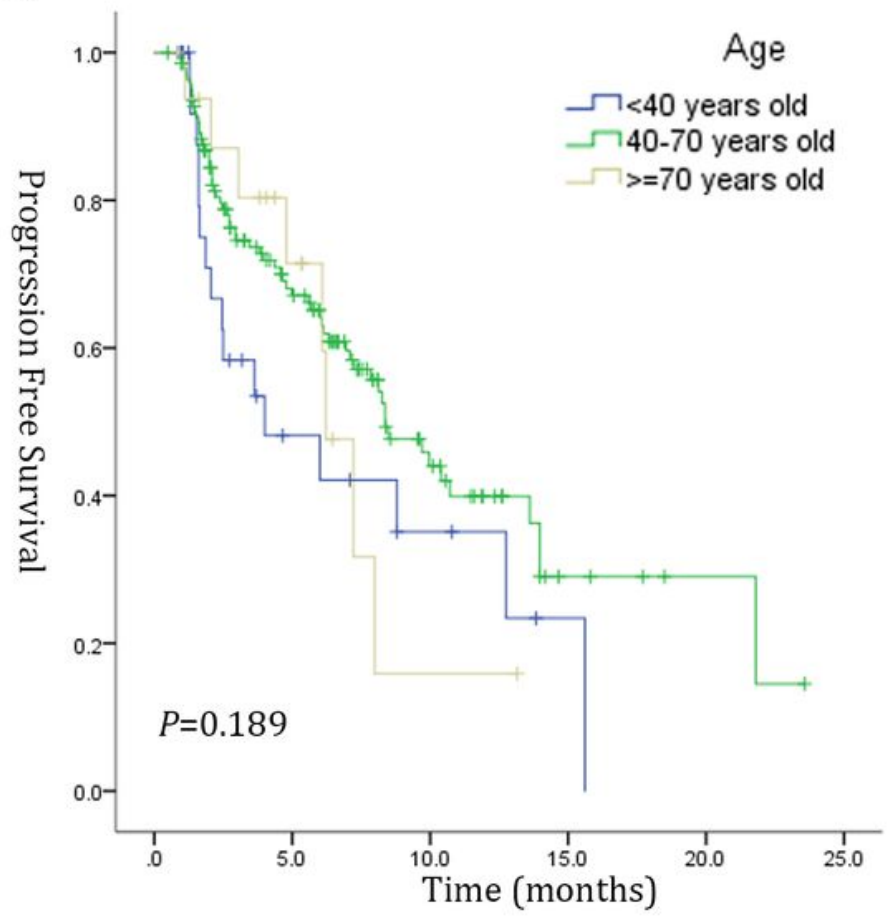

(C)

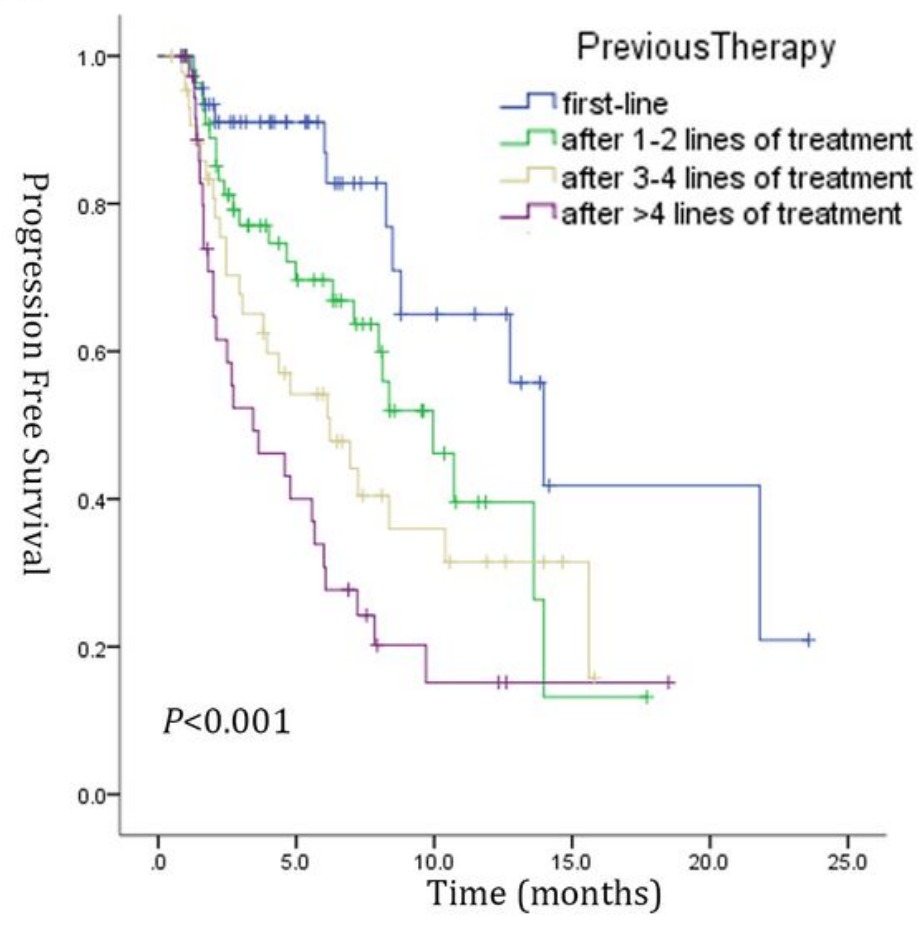

(B)

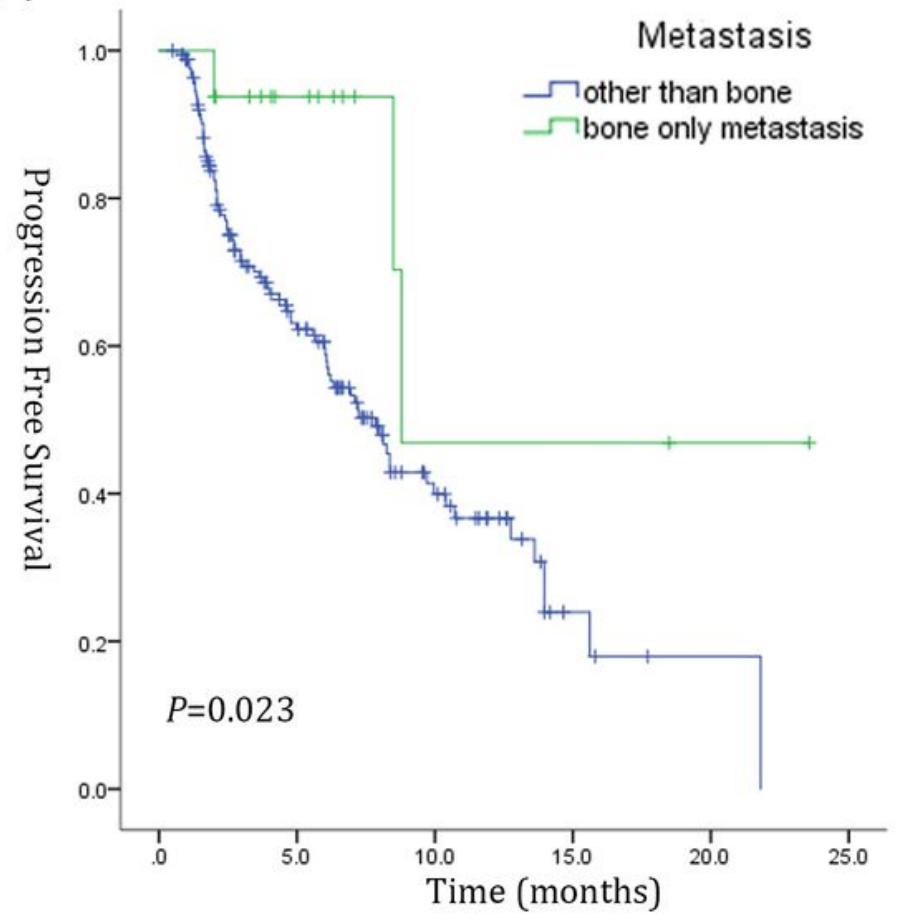

(D)

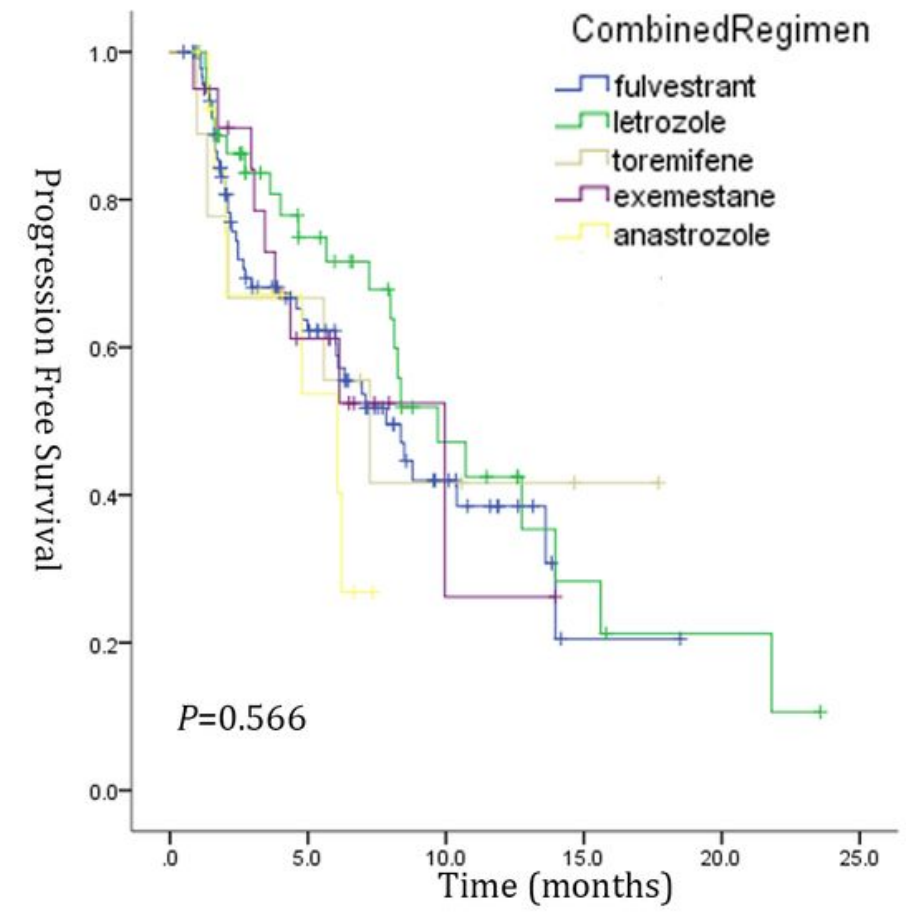

Figure 2

Kaplan-Meier curves revealing progression free survival according to age (A), metastatic site (B), previous lines of treatment (C), and various regimen combinations (D). 

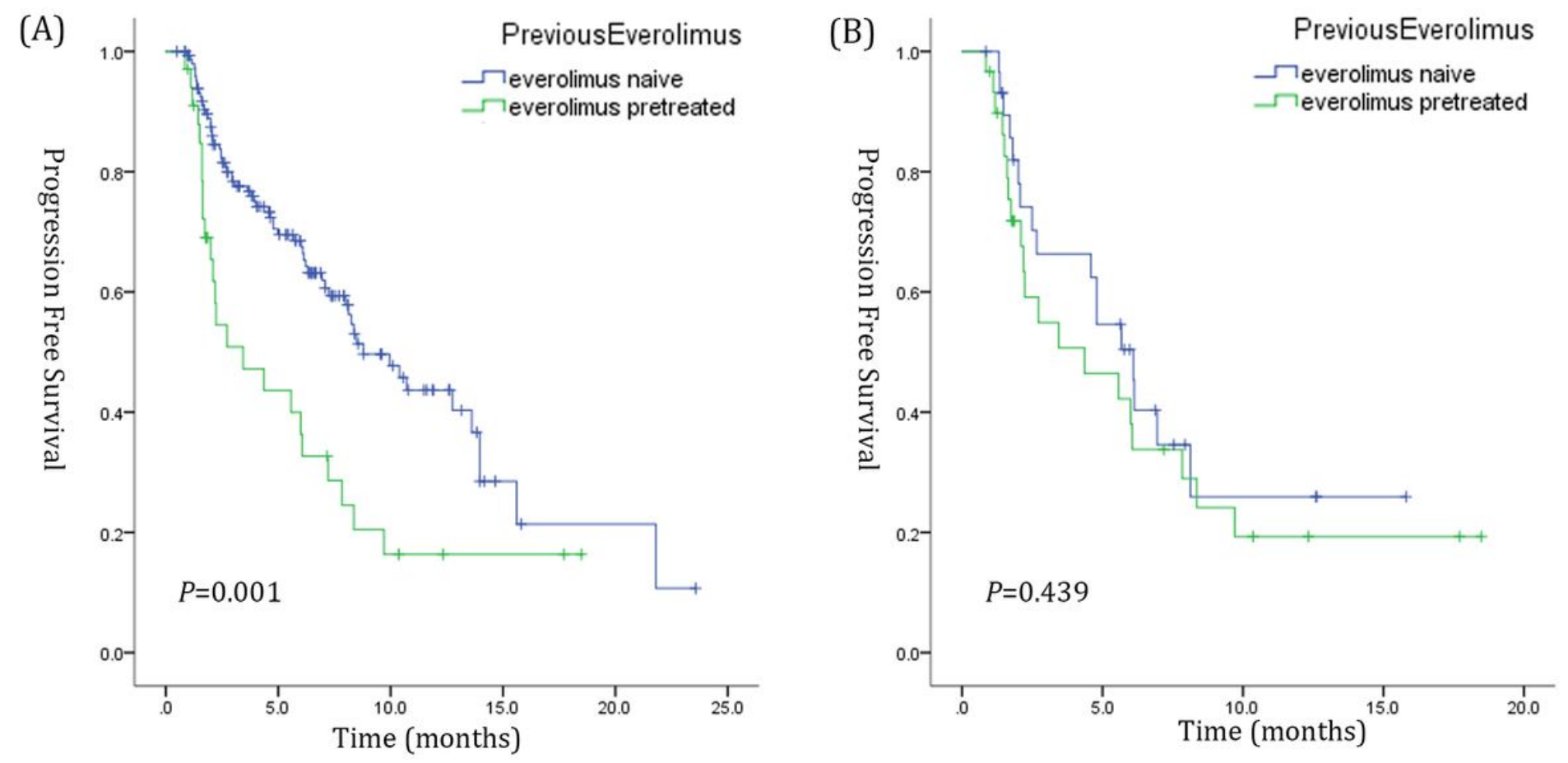

\section{Figure 3}

Kaplan-Meier estimates of progression free survival for various everolimus combinations in patients with metastatic breast cancer.

\section{Supplementary Files}

This is a list of supplementary files associated with this preprint. Click to download.

- coverletter0407.docx 